\title{
Chemotherapie wieder im Aufwind
}

In den letzten Jahren konnte man durchaus den Eindruck gewinnen, dass die konventionelle Chemotherapie durch die Immunonkologie verdrängt werden würde. Unbestritten haben die Immunonkologie und die Präzisionsmedizin phantastische Fortschritt für die Patienten erzielt. Trotzdem erweisen sich Prognosen zum Beispiel zur abnehmenden Bedeutung der Chemotherapie als vorschnell. Gerade die neuesten Studienergebnisse z. B. vom ASCO 2018 weisen darauf hin, dass die Kombination einer immunonkologischen Substanz mit einer konventionellen Chemotherapie sehr gute, vielleicht sogar synergistische Ergebnisse liefern. Für das nichtkleinzellige Bronchialkarzinom beispielsweise belegen mehrere Studien, dass eine Kombinationstherapie von Chemo- und Checkpointinhibitoren durchaus einen Vorteil für das Gesamtüberleben bringen kann. Monokausale Erklärungen für die Wirkungsweise der immunonkologischen Therapie reichen in der Regel nicht aus, daher wäre eine noch engere Verzahnung von Grundla- gen- und klinischer Forschung sehr wünschens- bzw. erstrebenswert.

Unser Ziel ist, diese klinischen, sprich für die Patienten relevanten Fortschritte, in der best practice onkologie vorzustellen und damit in die onkologische Versorgung zu transportieren.

Mit den besten Grüßen verbleibt

Ihr

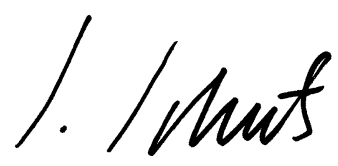

Prof. Dr. Stephan Schmitz

Schriftleiter von best practice onkologie

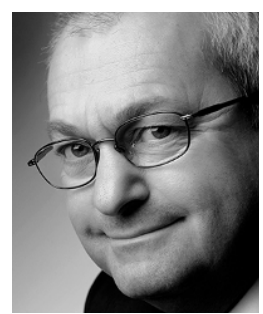

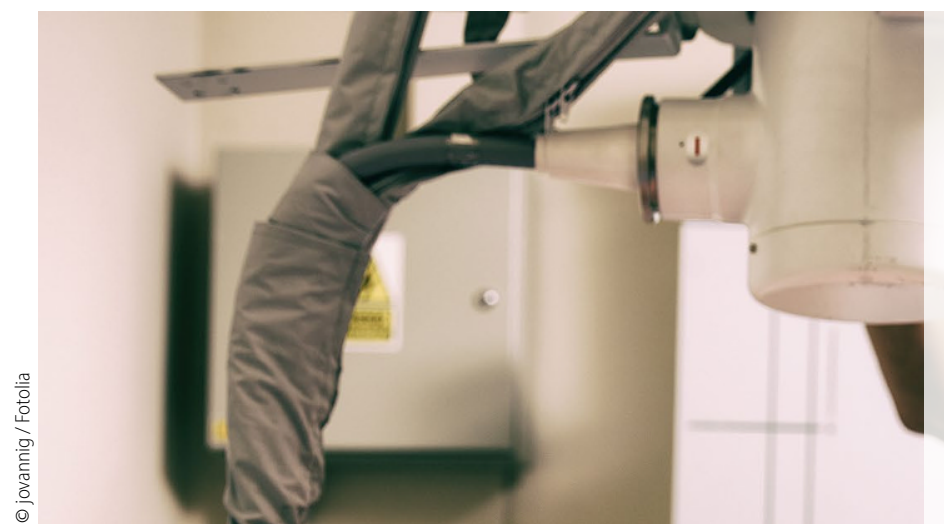

CME-TOPIC: Supportive Maßnahmen in der Radioonkologie Die Strahlentherapie ist ein integraler Bestandteil der Tumortherapie und wird häufig mit Systemtherapie und lokalen operativen Therapieverfahren, simultan oder sequentiell, kombiniert. Daher ist es auch wichtig, mögliche radiogene Nebenwirkungen zu verstehen, zu therapieren und bestenfalls vorzubeugen. Lesen und lernen Sie daher mehr über Radiodermatitis, orale Mucositis, Osteoradionekrose, Xerostomie, Enteritis, Proktitis, Pneumonitis, Nausea/Emesis und über die Nebenwirkungen im ZNS. 